reduced dihydoxyphenylglycol(DHPG) levels, and high ratios of DOPA:DHPG and DOPAC:DHPG. In contrast to patients with absent DBH, norepinephrine (NE) levels were normal in 4 Menkes patients and in the CSF of all 10 patients. The pattern was consistent with partial deficiency of DBH activity and compensatory increases in catecholamine biosynthesis in sympathetic nerves and brain. The changes may provide a biochemical marker for Menkes disease. (Kaler SG et al. Plasma and cerebrospinal fluid neurochemical pattern in Menkes disease. Ann Neurol Feb 1993; 33: 171-175). (Correspondence: Dr Kaler, National Inst of Health, Bldg 10, Rm 9S 242, 9000 Rockville Pike, Bethesda, MD 20892).

COMMENT. Menkes disease (Kinky-Hair disease) is a sex-linked recessive, neurodegenerative disorder of gray matter involving copper metabolism. Clinically, it is characterized by failure to thrive, cherubic facies, twisted and fractured hair, seizures, hypotonia, and progressive psychomotor deterioration. Biochemically, serum copper and ceruloplasmin levels are reduced or low-normal, and copper uptake by cultured fibroblasts is abnormally increased, permitting intrauterine diagnosis of the disease. Copper levels in liver and brain are low, but are high in the intestine and kidney. The incorporation of copper into enzymes such as DBH requiring this cofactor is impaired. DBH activity was assessed in the present study by measuring levels of DA, NE, and metabolites to provide a picture of catecholamine synthesis and turnover in infants with Menkes disease.

\title{
NEUROPATHIES
}

\section{PROGRESSIVE MONONEUROPATHY}

Six patients, aged 10 to 27 years, with insidiously progressive, painless weakness in the distribution of a single major lower extremity nerve are reported from the University of California, San Francisco. The duration at diagnosis varied from 3 months to 8 years. The sciatic nerve was involved in three patients, the common peroneal in two, and the femoral nerve in one. The appearance of the nerve at surgery was normal in two, atrophied in two, and fibrosed in one. EMG revealed a chronic axonal mononeuropathy without conduction block or focal conduction slowing. MRI, CT, and US imaging failed to reveal a nerve mass or compression. (Engstrom JW et al. Idiopathic, progressive mononeuropathy in young people. Arch Neurol Jan 1993; 50: 2023). (Reprints: Dr Engstrom, Box 0114, M794, Department of Neurology, University of California, San Francisco, CA 94143).

COMMENT. The authors ask the question: Could the patients have had a nerve tumor not uncovered by standard MRI or surgery and too

proximal for localization by nerve conduction studies? The diagnosis of 
neurofibromatosis type 2 was confirmed by a gadolinium MRI in a 14year-old girl who presented with flaccid weakness and wasting of the left upper limb and EMG evidence of brachial plexopathy (Millichap JG, Gomez MR. Ped Neur Briefs July 1990; 4 : 50-51). The standard MRI was inconclusive whereas the gadolinium imaging revealed enlargement of neural formina C 5-6 on the left side and evidence of a plexiform neurofibroma. Examination of the skin showed depigmented and cafeau-lait patches, helpful in the diagnosis in this case.

\section{CRITICAL-ILLNESS POLYNEUROPATHY}

A review of the neurological complications of sepsis from the University of Western Ontario, London, and Queen's University, Kingston, Ontario, Canada, draws attention to "critical-illness polyneuropathy" as a cause of difficulty in weaning from the ventilator. Patients with major medical, surgical, or traumatic illnesses who are in the recovery phase of septic encephalopathy may develop respiratory difficulties as an early sign of polyneuropathy. Electrophysiological studies, showing reduction of diaphragmatic action potential and denervation of chest wall muscles, may be diagnostic of the neuropathy before weakness of limbs and areflexia develop. In contrast to the central nervous system, damage to the peripheral nervous system is more severe and sometimes persistent in the septic syndrome. (Bolton CF et al. The neurological complications of sepsis. Ann Neurol Jan 1993; 33: 94-100). (Correspondence: Dr Bolton, Victoria Hospital, 375 South Street, London, Ontario N6A 4G5, Canada).

COMMENT. In addition to encephalopathy and polyneuropathy, patients with sepsis may develop myositis or myopathy and an elevated CPK. The EEG and EMG are sensitive indicators of these complications of the septic syndrome.

\section{CEREBROVASCULAR DISEASE}

\section{ASYMPTOMATIC ANEURYSMS AND HIV INFECTION}

Aneurysms of major cerebral arteries in two children, aged 11 and 12 years, infected with human immunodeficiency virus from blood transfusions are reported from the National Institutes of Health, Bethesda, MD, and Georgetown University Hospital, Washington, DC. The incidence among 250 children treated and monitored with MRI was 0.8 percent. The aneurysms developed and progressed during therapy and follow-up but were asymptomatic. The role of HIV infection in these lesions was undetermined. (Husson RN et al. Cerebral artery aneurysms in children infected with human immunodeficiency virus. LPediatr Dec 1992; 121: 927-930). (Reprints: Philip A 